\title{
XXIV.
}

\section{Castration oder Resection des Nebenhodens bei Epididymitis tuberculosa?}

\author{
Von \\ Dr. Otto Lanz, \\ Docent für Chirurgie in Bern.
}

(Mit 2 Abbildungen.)

Die Principien der conservativ en Chirurgie sind dank der aseptischen Wundbehandlung und der Vervollkommnung unserer Resectionstechnik so sehr ins chirurgische Denken und IIandeln übergegangen, dass auch der besehäftigte Operateur nicht jedes Semester mehr zu einer Amputation sich gezwungen sieht. Ausnahme von dieser Regel macht wenigstens bei der Mehrzahl der deutschen Chirurgen der II oden. Während bei tuberculüser Epididymitis von den franzüsischen Chirurgen der blossen Resection tuberculös erkrankter Theile des Nebenhodens das Wort geredet wird, neigt man von deutscher Seite mehr dazu, partielle Operationen im Hinblick auf die häufige Mitbetheiligung des Hodens zu verwerfen und als Normalverfahren die einfachere Castration vorzuziehen. Gegen die principielle $\Lambda \mathbf{n}$ wendung der Castratio spricht auf der anderen Seite die wohl ebenso häufige Mitbetheiligung anderer Urogenitalorgane, der Lmstand, dass die primäre Hodentuberculose eine seltene Ausnahme ist. Die Geschlechtsdrüsen scheinen überhaupt der Ansiedelung des Tuberkelbacillus fast ebenso ungünstigen Boden zu bieten wie die Schilddrüse; bei Miliartuberculose bleibt der Hoden fast stets frei und auf Traumen, die denselben gemäss seiner exponirten Lage so oft treffen, reagirt er mit einer Hydrocele, viel seltener mit Tuberculose. Indem nun die tuberculöse Iocalisation auf den Hoden in vielen, wenn nicht vielleicht den meisten Fällen, ihren Ausgangspunkt von einer anderen Stelle des Lrogenitaltractus, von Prostata, Blase oder Niere nimmt, ist die doppelseitige Erkrankung ein relativ häufiges Vorkommniss. Eine beidseitige gleichzeitige tuberculöse Erkrankung der Hoden 
ist ungemein selten, vielmehr entwickelt sich eine solche stets consecutiv, so dass man nach einseitiger Castration sich des Oefteren gezwungen sieht, in einem zweiten Act auch den anderen Testikel wegzunehmen.

Es wäre deshalb die Schonung des Hodens - wenn einwandsfrei möglich - ein sehr wichtiger Factor, da man sich nicht damit trösten kann, der zweite Testikel bleibe ja intact, und da die doppelseitige Castration beim Manne einen äusserst fatalen Eingriff vorstellt, wenigstens so lange männliche Chirurgen das Messer führen.

Die Tuberculose der männlichen Geschlechtsdrüsen tritt fast ausnahmslos als Epididymitis, nicht als Orchitis auf, was ja bekanntlich als eines der wichtigsten Momente für die Diagnose gilt. Kommen die Patienten in späteren Stadien der Erkrankung zum Arzte, so ist die Frage, ob der Hoden intact oder ebenfalls tuberculös erkrankt ist, meist leicht zu entscheiden; in den ersten Monaten der Krankheit ist aber die Mitbetheiligung des Hodens klinisch in den meisten Fällen nicht festzustellen.

Bei einem 15 jährigen Jungen, den ich im Februar 1896 an der Kocher'schen Klinik wegen tuberculöser Epididymitis operirte, machte ich daher zum ersten Mal eine Resectio testis in folgender Weise: Der Nebenhoden hatte in der Cauda einen wallnussgrossen tuberculösen Knoten mit Abscess, einige kleinere Knoten gegen das Caput zu; der Hoden schien völlig normal und ebenso das Vas deferens. Aus den oben entwickelten Grïnden schien mir ein Vortheil darin zu liegen, den Testikel zu erhalten, für den Fall, dass er völlig gesund war. Der Palpation nach schien er es zu sein; um jedoch möglichste Sicherheit darüber zu gewinnen, spaltete ich nach exacter Resection der ganzen Epididymis den Hoden median, gleichwie man eine Niere bei der Section spaltet, klappte ihn auf und unterwarf die Schnittflächen einer genauen Inspection. In der Hodensubstanz war absolut nichts zu entdecken, was Verdacht erweckt hätte; deshalb klappte ich das Organ wieder zusammen und vernähte die Tunica albuginea mit einer fortlaufenden Catgutnaht. Das Vas deferens schien bei Palpation und Inspection etwas verdickt, weswegen es möglichst hoch hinauf resecirt wurde; aufgeschnitten zeigte es indes keine Veränderungen. Der Patient verliess das Bett 2 Tage später mit einem Suspensorium, der Hoden war Anfangs etwas gesch wollen, druckempfindlich und zeigte namentlich am oberen Pol Infiltrationen, die sich aber schon nach 14 Tagen zurückgebildet hatten. Bei einer Untersuchung: 2 Monate später erschien der Hoden von gleicher Grösse wie auf der rechten Seite, doch etwas schlaffer. An Stelle des Nebenhodens eine 
narbige, derbe, nicht druckempfindliche Schwiele. Die specifische Empfindlichkeit des Testikels auf Druck völlig intact. Hoden völlig frei beweglich, mit der Haut nicht verwachsen. Samenstrang bis auf das fehlende Vas deferens normal, die Arterie fühlbar. - 11/2 Jahre später wurde die andauernde Heilung constatirt.

Seither habe ich mehrfach wegen tuberculöser Epididymitis operativ eingreifen müssen; entweder war aber die Mitbetheiligung des Hodens von vornherein klar, oder aber es bestanden Fisteln, welche mir eine Complication der Castration nicht räthlich erscheinen liessen. Einzig in meinem letzten Falle sah ich mich wieder zu der Aufklappung des Testikels veranlasst, indem bei intensiver Erkrankung der Epididymis der Hoden bei der Palpation und Inspection vor und während der Operation völlig normal, gleich gross und von gleicher Consistenz wie sein gesunder Partner zu sein schien.

Ich lasse diese Beobachtung als Parallelfall $\mathrm{zu}$ dem oben erwähnten sammt Abbildung der gewonnenen Präparate hier folgen:

Herr P. aus K., 33 Jahre alt, sonst stets gesund, machte vor 15 Jahren einen Misstritt mit dem rechten Fuss, der unterbalb des Malleolus externus anschwoll. 11 Monate später musste daselbst eine Incision gemacht werden; Pat. kann aber keine Angaben machen, welche die tuberculöse Natur dieses Leidens sicherten. - Anfangs December 1899 stellten sich nach und nach zunehmende Schmerzen in der rechten Inguinalgegend ein. Beim Nachfühlen constatirte er, dass der rechte Hoden geschwollen war und der consultirte Arzt diagnosticirte eine Nebenhodenentzündung, verordnete ein Suspensorium, Jodanstriche und später Ungt. cinereum. Unter dieser Behandlung ging die Schwellung anfänglich deutlich zuritck, der Nebenhoden wurde derber, massiver und im Januar hatte Pat. fast keine Schmerzen mehr. Allein jetzt im Laufe des Februar hat die Anschwellung wieder etwas zugenommen und die Schmerzen haben sich wieder eingestellt, was den behandelnden Arzt veranlasst, den Chirurgen beizuziehen. Von Vaterseite stammt Pat. aus sehr kräftiger Familie, von mütterlicher Seite dagegen ist er tuberculös belastet. Trauma oder Gonorrhoe werden in Abrede gestellt.

23. Februar 1900. Status: Kräftig gebauter Mann von gesunder Gesichtsfarbe und trefflich entwickelter Musculatur. Brust- und Bauchorgane keine Veränderungen. Der rechte Testikel stellt einen kleinhühnereigrossen Tumor dar und die Palpation ergiebt, dass die Anschwellung einzig dem Nebenhoden zur Last fällt. Derselbe weist die Dicke einer Bratwurst auf, ist von unregelmässig-knolliger Oberfäche, gleichmässig derber Consistenz, druckempfindlich. Der Hoden selbst erscheint, mit der linken Seite verglichen, in Grösse und Consistenz, absolut vormal; das Vas deferens in seinem unteren Antheil etwas verdickt. Von Seiten der Nieren, der Blase und der Prostata keine Veränderungen nachweisbar.

24. Februar. Operation in leichter Aethernarkose. Inguinalschnitt und Freilegung des Samenstranges über dem vorderen Leistenring. 
Nach Isolirung wird versucht, den Hoden in seinen Hüllen empor zu luxiren; er folgt aber dem Zuge anfänglich nicht, obschon bei der Untersuchung keine Beziehungen zur Haut nachgewiesen worden waren. Ein haselnussgrosser, eitrig-gelb durchschimmernder Höcker der Epididymis hatte sich bereits zur Perforation angeschickt und war dementsprechende Verwachsungen eingegangen. Nach Lösung derselben mit dem Messer macht sich die Luxation ohne Weiteres. Der Nebenhoden ist in toto intensiv erkrankt, der Hoden dagegen zeigt auch bei der Inspection nicht die leichteste Veränderung. Es wird deshalb in Aussicht genommen, den

Fig. 1.

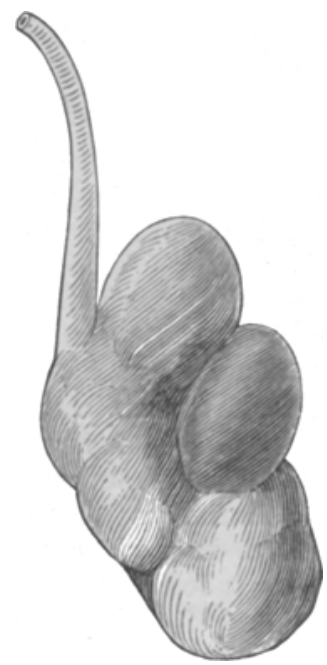

Fig. 2.

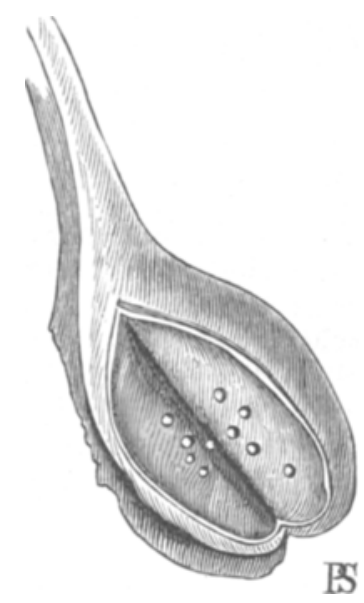

Testikel womöglich zu erhalten und in einem ersten Act mit Messer und Pincette die Epididymis sehr exact und sauber unter möglichster Schonung der zum Hoden ziehenden Gefässe excidirt, ohne jede Schwierigkeit vom Hoden gelöst, das Vas deferens möglichst weit aus der Bauchhöhle hervorgezogen und hoch resecirt; dasselbe zeigt keine Veränderungen.

Aus diesem präparatorischen Vorgehen resultirt das Präparat (Fig. 1). Dem gelblich verfärbten, adhärent gewesenen Knoten entspricht auf dem Durchschnitt ein grösserer Eiterherd, den stärkeren Verdickungen an Caput und Cauda liegen ansgedehnte Verkäsungen zu Grunde.

Nun steht also der in seinen Gefässverbindungen so weit thunlich geschonte Hoden noch in möglichst intactem Zusammenhange mit dem Samenstrang, aus dem nur das Vas deferens herausgelöst worden ist. Um aber sicher zn gehen, dass mit der Schonung des Hodens keine tuberculösen Herde zuräckgelassen werden, soll er in seinem Längsdurchmesser aufgeschnitten werden; zeigt er sich frei, so wird er wieder zusammengeklappt und durch eine fortlaufende Naht der Albuginea goschlossen. Da dies nicht der Fall ist, sondern die Schnittfäche eine 
Reihe von Tuberkelknötchen aufweist, wird die Castration angeschlossen, wodurch das Präparat (Fig. 2) gewonnen wird. - Die fortlaufende Hautnaht wird am 27. Februar entfernt und 3 Tage später verlässt Pat. das Spital.

Der eben mitgetheilte Fall beweist, dass der Hoden schon ganz im Beginn der Erkrankung ergriffen und dass es ganz unmöglich sein kann, seine Mitbetheiligung auszuschliessen. Ich halte deshalb die partiellen Operationsverfahren nicht für rationell, es werde denn der Resection der Epididymis eine A u topsie des Hodens hinzugefügt. In welchem Maasse das seiner Helmzier beraubte Organ durch seine „innere Secretion" dem Körper noch Dienste zu leisten, die Psyche noch zu beeinflussen vermag, wage ich allerdings nicht zu entscheiden. Auch liegt es auf der Hand, dass die makroskopische Besichtigung einer Schnittfläche keine absolute Garantie bietet.

Mit grösserer Berechtigung als bei der einseitigen Castration wird man von meinem Operationsverfahren Gebrauch machen in Fällen, wo es sich um den zweiten Hoden handelt. Hier wird man eventuell sogar in die Lage kommen, einen Hoden mit Hülfe von Thermokauter und Jodoformeinschichtung zu erhalten, selbst wenn ein oder das andere Tuberkelknötchen in die Hodensubstanz eingesprengt ist.

Aufgefallen ist mir in meinen letzten Fällen von Epididymitis tuberculosa, dass sich von 7 Beobachtungen fünf auf die rechte Seite bezogen; ob dies ein Zufall ist, oder ob es damit zusammenhängt, dass die Vena spermatica interna rechts direct zur Vena cava inf. emporgeht, während sie links unter rechtem Winkel in die Vena renalis einmündet, ob sich also diese Beobachtung im Sinne der Bier'schen Staungshyperämie verwerthen liesse, müssten grosse Beobachtungsreihen lehren. 